\title{
EEG als Steuersignal: Gehirnaktivität entschlüsseln und effizient als Kommunikationsmittel für Patienten mit motorischen Defiziten nutzen
}

\section{EEG as a Control Signal: Decoding of Brain Activity and its Efficient Application as Communication Channel for Patients with Motor Deficits}

Autoren

Christoph Reichert11, 2, 3, Stefan Dürschmid11, 4, Hermann Hinrichs 1, 2, 3, 4

Institute

1 Abteilung Verhaltensneurologie, Leibniz-Institut für Neurobiologie, Magdeburg

2 Center for Behavioral Brain Sciences (CBBS), Magdeburg

3 Forschungscampus STIMULATE, Magdeburg

4 Klinik für Neurologie, Universitätsklinikum Magdeburg

\section{Schlüsselwörter}

räumlicher Filter, event-korreliertes Potenzial, Gehirn-Computer Schnittstelle

Key words

Spatial filter, event-related potential, brain-computer interface

Bibliografie

DOI https://doi.org/10.1055/a-1135-3782

Online-Publikation: 23.7.2020

Klin Neurophysiol 2020; 51: 161-166

(c) Georg Thieme Verlag KG Stuttgart · New York

ISSN 1434-0275

Korrespondenzadresse

Dr. Christoph Reichert

Leibniz-Institut für Neurobiologie

Brenneckestraße 6

39118 Magdeburg

christoph.reichert@lin-magdeburg.de

\section{ZUSAMIMENFASSUNG}

Ziel der Studie Ereignis-korrelierte Potenziale werden in der Regel in einzelnen EEG-Kanälen ermittelt. Mit einem einzelnen Kanal wird jedoch nur ein Teil des gesamten Hirnprozesses erfasst. Für eine Gehirn-Computer Schnittstelle, die in kurzer Zeit eine Entscheidung treffen muss, ist diese singuläre Gehirnantwort häufig unzureichend wogegen die Information aus mehreren Kanälen häufig redundant ist. Beide Vorgehensweisen sind nicht optimal. Daher ist es unser Ziel, die Kanäle zu wenigen Komponenten zu kombinieren, die die relevantesten Modulationen eines Hirnprozesses erfassen.
Methodik Wir nutzen die kanonische Korrelationsanalyse, um datengetrieben räumliche Filter aus dem EEG zu bestimmen. Mit der Produkt-Moment Korrelation ermitteln wir, auf welche von 12 verschiedenen Stimulussequenzen die Studienteilnehmer geachtet haben.

Ergebnisse Die verdeckte Aufmerksamkeit der Studienteilnehmer konnte mit hoher Genauigkeit (89,3 $\pm 9,2 \%$ ) aus dem räumlich gefilterten EEG und signifikant besser als aus einzelnen Kanälen dekodiert werden.

Schlussfolgerung Die aus dem EEG erlernten räumlichen Filter ermöglichen die Extraktion von Komponenten, die einen event-korrelierten Gehirnprozess charakterisieren und eine Gehirn-Computer Schnittstelle effektiv steuern können, was von hoher Relevanz für Patienten ist, die nicht mehr anderweitig kommunizieren können.

\section{ABSTRACT}

Objective Event-related potentials are commonly calculated in single EEG channels. However, a single channel reflects only a part of the whole brain process. A brain-computer interface must detect a user's intention in short intervals and thus this sparse brain response might be insufficient while information in multiple channels might be redundant. Therefore, our aim was to combine the channels to a few components which reflect the most relevant modulations of a brain process.

Methods We applied canonical correlation analysis to determine spatial filters from EEG signals. Using an ordinary correlation measure, we decoded to which of 12 stimulus sequences the participants directed their attention.

Results The covert attention of participants was decoded i) with high accuracy $(89,3 \pm 9,2 \%)$ from spatially filtered EEG signals and ii) significantly better compared to using single channel features.

Conclusions The spatial filters, learned from EEG data, permitted the extraction of components characterizing an eventrelated brain process. Furthermore, they could be effectively used to control a brain-computer interface, which is highly relevant for patients who have lost all other communication abilities. 


\section{Einleitung}

Hirn-Computer Schnittstellen (engl.: brain-computer interfaces; $\mathrm{BCl}$ ) [1] haben das Potenzial, eine Kommunikation mit Patienten zu ermöglichen, die eigentlich aufgrund ihrer schweren motorischen Beeinträchtigungen nicht mehr in der Lage dazu sind. BCls werden häufig realisiert, indem verschiedene visuelle Reize präsentiert werden, um zu erfassen, was Patienten ausdrücken wollen. Durch das willentliche Ausrichten von Aufmerksamkeit auf einen bestimmten Reiz werden im Gehirn spezifische ereigniskorrelierte Potenziale (EKPs) generiert, die vom BCI detektiert werden. Problematisch könnte es dabei sein, dass diese EKPs erst durch viele Messwiederholungen und Mittelung detektiert werden können, wobei die schnelle Steuerung eines BCls eher auf wenigen zur Verfügung stehenden Daten beruht. Die zuverlässige Dekodierung aus dem EEG ist daher schwierig, denn das Signal einer Einzelmessung ist schwach im Vergleich zum Rauschen. Hinzu kommt, dass EKPs theoretisch räumlich und zeitlich gut definiert sind, praktisch aber nicht zwingend klar ist, in welchem Kanal man welche typische Veränderung des Oberflächenpotenzials bei individuellen Probanden erwarten kann. Für die Dekodierung ist es aber wichtig, möglichst informative Merkmale aus dem EEG zu extrahieren und redundante sowie irrelevante Merkmale zu verwerfen. Häufig werden die Merkmale hypothesengetrieben selektiert. Beispielsweise werden bekannte Charakteristika der P300 angewandt, um den EEG-Kanal und das Intervall zu definieren, in denen ein Unterschied erwartet wird, wenn ein Nutzer auf einen selten präsentierten Zielstimulus achtet. Möglicherweise ist aber das EKP komplexer und enthält weitere Charakteristika, die zu einer verlässlicheren Dekodierung führen. Daher wird häufig eine Extraktion von Merkmalen bevorzugt, die es erlaubt, aus den Daten zu lernen, welche Merkmale für die Dekodierung informativ sind. Beispielsweise gibt es verschiedene Ansätze, individuelle Kanäle zu selektieren, die vielversprechend für die Dekodierung sind [2-4].

Ein anderer Ansatz, die Anzahl der Kanäle zu reduzieren, ist die Verwendung von räumlichen Filtern. Dabei werden die Kanäle so gewichtet, dass die Linearkombination der gemessenen Werte in den Kanälen, also die Summe der gewichteten Messwerte, den Wert eines neuen, virtuellen Kanals ergibt. Diese Vorgehensweise bringt verschiedene Vorteile. I) Relevante Kanäle bekommen ein hohes Gewicht, unrelevante Kanäle bekommen sehr kleines Gewicht, was einer Kanalselektion ähnelt. II) Common-mode Noise (Rauschen, das in allen Kanälen auftritt) kann durch entgegengesetzte Gewichtung unrelevanter Kanäle entfernt werden. III) Potenziale, die sich über mehrere Kanäle verteilen, was bei Oberflächen EEG durchaus üblich ist, können durch Superposition mehrerer Kanäle verstärkt werden, bzw. kann unspezifische Aktivität wie Rauschen unterdrückt werden. IV) Ein einzelner räumlicher Filter, also eine Kombination von Kanalgewichtungen reduziert das gesamte EEG auf einen Kanal. Üblicherweise werden einige wenige solcher, voneinander verschiedene Kanalgewichtungen angewandt, was die Anzahl der Kanäle deutlich reduziert. Ein Beispiel für einen räumlichen Filter ist der Laplace Filter, bei dem das Signal eines Kanals lokal verstärkt wird, indem benachbarte Kanäle negativ gewichtet werden, d. h. jeder Kanal wird durch einen eigenen räumlichen Filter bereinigt.

Die Schwierigkeit, mithilfe von räumlichen Filtern ein möglichst informatives Signal für eine Detektion zu extrahieren, ist, eine op- timale Gewichtung aller Kanäle zu bestimmen. Häufig kommen dafür Verfahren der Stochastik zum Einsatz. Ein Beispiel ist die in $\mathrm{BCl}$ Anwendungen häufig verwendete Common Spatial Pattern (CSP) Methode, bei der eine Gewichtung der Kanäle bestimmt wird, die die Varianz unter einer ersten Experimentalbedingung maximiert und gleichzeitig für eine zweite Experimentalbedingung minimiert (für Details siehe [5]) und sich besonders für die Detektion von motorischer Vorstellung bewährt hat, die häufig für die Steuerung von $\mathrm{BCls}$ verwendet wird [6].

In diesem Artikel stellen wir ein Verfahren vor, mit dem räumliche Filter anhand gemessener EEG Daten bestimmt werden, die wir dann für die Detektion von EKPs verwenden. Wir zeigen, dass mit diesem Verfahren eine hohe Genauigkeit bei der Dekodierung von spezifischer Information im EEG, die für die Steuerung eines BCls notwendig ist, erzielt werden kann. Außerdem ist das Verfahren geeignet, aus beliebigen anderen EKP Untersuchungen datengetrieben differentielle EEG Komponenten zu extrahieren, was für eine Anwendung im klinischen Kontext von großem Interesse ist.

\section{Material und Methoden}

\section{Datenakquise}

Die EEG-Daten für die hier gezeigte Analyse wurden simultan in einer MEG (Magnetoenzephalografie) Studie erhoben, in der $19 \mathrm{ge}-$ sunde Probanden teilnahmen. Die Aufgabe der Probanden war es, eines von 12 mit gleichem Abstand zu einem Fixationspunkt angeordnete Objekte zu beachten, wobei Augenbewegungen vermieden werden sollten. Die Objekte wurden mit visuellen Stimuli in zufälliger Reihenfolge hervorgehoben, was dazu führt, dass bei visueller Stimulation des Objektes, das beachtet wird, als typisches EKP eine P300 hervorgerufen wird. Dieses wird in unserer Studie anhand von Magnetfeldänderungen über dem Kopf detektiert. Das P300 Signal, welches bei Präsentation des Zielstimulus eine erhöhte Amplitude aufweist, wird häufig zur Steuerung von BCls genutzt [7]. Der Ablauf des Experiments ist in > Abb. 1 dargestellt. Eine detailierte Beschreibung findet sich in [8].

Das EEG wurde nach einem erweiterten 10-20 System mit insgesamt $29 \mathrm{Ag} / \mathrm{AgCl}$-Elektroden abgeleitet und mit einem EPA-6 Verstärker der Firma Sensorium bei einer Abtastfrequenz von $508,63 \mathrm{~Hz}$ digitalisiert. Alle Kanäle wurden gegen Mittelwert des rechten und linken Mastoiden referenziert. Die Impedanzen wurden unter $5 \mathrm{k} \Omega$ gehalten. Außerdem wurde ein horizontales/vertikales EOG (Elektrookulogramm) aufgezeichnet, um Augenbewegungen zu überwachen.

\section{Datenvorverarbeitung}

Die EEG Daten wurden mit einem FIR Bandpassfilter $(0,25-15 \mathrm{~Hz})$ gefiltert und die Abtastrate nach der Aufzeichnung auf $254,3 \mathrm{~Hz}$ reduziert. Anschließend wurden Epochen mit einer Dauer von 800 ms, beginnend nach Präsentation eines Stimulus extrahiert. Eine Epoche ist demnach eine Matrix der Größe $n \times k$, wobei $n$ die Anzahl der Abtastpunkte innerhalb des Analyseintervalls und $k$ die Anzahl der Kanäle ist. In jedem Trial wurde jedes der 12 Objekte genau 5-mal durch einen Strimulus (kurzzeitiges Aufhellen des Hintergrundes) hervorgehoben. Epochen, die einem Stimulus des Objektes folgen, auf das der Proband seine Aufmerksamkeit gelenkt 


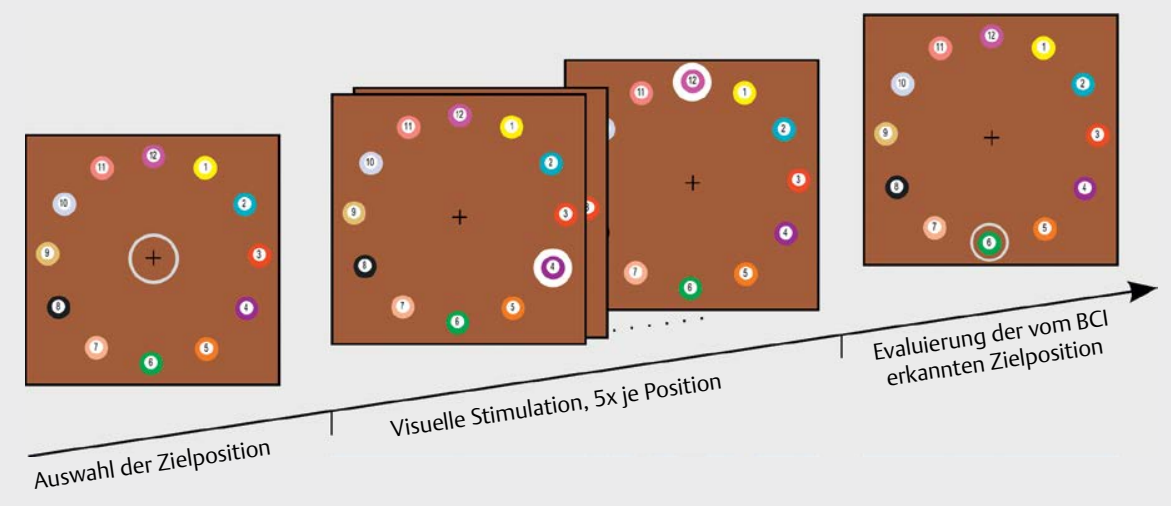

- Abb. 1 Die Studienteilnehmer haben am Beginn eines Trials selbst entschieden, welches der 12 Objekte sie mit ihrem Aufmerksamkeitsfokus wählen wollen. Anschließend wurde innerhalb von 10 Sekunden jedes Objekt einzeln in zufälliger Reihenfolge 5-mal mit einem visuellen Stimulus hinterlegt. An der vom BCl ermittelten wahrscheinlichsten Position wurde ein Ring angezeigt und die Richtigkeit des Feedbacks mittels Tastendruckes abgefragt. Bei falschem Feedback wurde die Abfrage mit der nächstwahrscheinlichsten Position fortgesetzt, bis die eigentliche Zielposition ermittelt wurde.

hat, nennen wir Targetepochen, alle anderen Standardepochen. Um ein ausgeglichenes Verhältnis der Anzahl von Target- und Standardepochen zu erreichen, beziehen wir nur die Standardepochen ein, die dem Objektstimulus gegenüberliegend des Zielreizes folgen.

\section{Bestimmung optimaler räumlicher Filter}

In einem datengetriebenen Dekodierungsansatz wollen wir eine optimale Gewichtung der EEG Kanäle finden, um die Unterschiede zwischen Standard- und Targetepochen möglichst effektiv aus einer Menge Trainingsdaten zu lernen. Dafür verwenden wir die kanonische Korrelationsanalyse (engl.: canonical correlation analysis; CCA) [9]. Dieses statistische Verfahren, analysiert den Zusammenhang zwischen 2 Variablenmengen $X$ und $Y$, indem Gewichtsvektoren $a$ und $b$ für die Variablenmengen derart bestimmt werden, dass die resultierenden Linearkombinationen $X a$ und $Y b$ maximal miteinander korrelieren. Die CCA wurde erstmals bei BCls eingesetzt, die darauf abzielen, Steady-state visuell evozierte Potenziale (SSVEPs) aus dem EEG zu dekodieren [10]. Hierfür bilden die EEG Daten die erste Variablenmenge und eine Kombination aus Sinusund Cosinusfunktionen, die die Stimulationsfrequenz modellieren, die zweite Variablenmenge. Die CCA kombiniert die Modellfunktionen so, dass eine Sinusschwingung mit an die SSVEPs angepasster Phase resultiert. Gleichzeitig werden Kanäle, die diese Schwingung enthalten hoch gewichtet und miteinander kombiniert, sodass die SNR erhöht wird. Sinusschwingungen sind für EKP Analysen jedoch wenig hilfreich, weshalb andere Modellfunktionen definiert werden müssen.

Wir nehmen an, dass der zeitliche Verlauf eines EKPs immer an einen festen Zeitpunkt, dem Präsentationsbeginn des Stimulus, gekoppelt ist. Wir legen fest, dass jedem abgetasteten Zeitpunkt einer Targetepoche eine eigene Modellfunktion zugeordnet wird, die diesen Zeitpunkt frei skalierbar macht. Setzen wir den Wert am $i$-ten Abtastpunkt einer Epoche in der $i$-ten Modellfunktion also 1, alle anderen Werte 0 . In Matrixschreibweise entspricht das der Einheitsmatrix $I_{n}$ der Größe $n$. Gleichermaßen setzen wir die Menge der Modellfunktionen für eine Standardepoche auf $-I_{n}$. Die Idee dabei ist, Signale aus Standardepochen gegen Signale aus Targetepochen zu gewichten und zu erreichen, dass Aktivierungen, die bei Standard und Targetpräsentation gleichermaßen auftreten, ignoriert werden. Um nun die Matrizen für die CCA zu generieren, verketten wir alle Epochen der Trainingsmenge zu einer Matrix $X$ mit $k$ Spalten und analog die zugehörigen Modellfunktionen zu einer Matrix $Y$ mit $n$ Spalten. Eine interessante Eigenschaft dieser Menge von Modellfunktionen ist, dass das Produkt $X^{T} Y$, gewichtet mit der Anzahl der Targetepochen, den Differenzwellen aus gemittelten Targetepochen und gemittelten Standardepochen entspricht. Analysieren wir $X$ und $Y$ nun mit einer CCA, bestimmt diese die Komponenten, die den Unterschied zwischen Standard- und Targetepochen am besten repräsentieren. Der Gewichtsvektor $a$, der $X$ linear kombiniert, entspricht einem räumlichen Filter, den wir im Sinne der Korrelation mit $Y b$ als optimal betrachten können. Die kanonische Komponente wird direkt durch den Gewichtsvektor $b$ repräsentiert, da dessen Linearkombination mit den Modellfunktionen der Targetepochen $I_{n} b=b$ ergibt. Die CCA ermittelt sukzessiv weitere Komponenten, bis eine der Variablenmengen keine Restvarianz mehr aufweist. Für das weitere Vorgehen der Dekodierung von EKPs benutzen wir nur Komponenten mit signifikanten kanonischen Korrelationen $(p<0,05)$, mindestens aber 2 Komponenten.

\section{Dekodierung mittels optimaler räumlicher Filter}

Die optimalen räumlichen Filter haben wir aus einer Menge von Trainingsdaten bestimmt. Nun werden die Gewichtsvektoren $a$ und $b$ auf EEG Daten angewandt, die nicht im Trainingsdatensatz enthalten waren. Mithilfe des resultierenden Signals ermitteln wir, auf welches von 12 Objekten der Proband seine Aufmerksamkeit gelenkt hat. Dafür werden alle 5 Epochen, die auf den Stimulus eines potenziellen Zielreizes innerhalb eines Trials folgen, miteinander zu einer Matrix mit $k$ Spalten verkettet und mit a multipliziert. Gleichermaßen wird die Menge der Modellfunktionen für Targetepochen $I_{n}$ fünffach zu einer Matrix mit $n$ Spalten verkettet. Anschließend wird die Produkt-Moment Korrelation der resultierenden Vektoren der Länge $5 n$ berechnet. Dies wird für alle Komponenten, die 
in der CCA das Signifikanzniveau erreicht haben, wiederholt und der Mittelwert aller Korrelationskoeffizienten bestimmt. Das Objekt, das den höchsten mittleren Korrelationskoeffizienten bewirkt, wird als Objekt des Zielstimulus klassifiziert.

Um die Performanz dieses Dekodierungsalgorithmus zu testen, bedienen wir uns der Kreuzvalidierung, bei der jeweils ein Durchgang (12 Trials) als Testdatensatz verwendet wird und alle anderen Durchgänge als Trainingsdatenmenge dienen.

\section{Ergebnisse}

Die Dekodierung der EEG Signale, die es ermöglicht zu bestimmen, auf welches von 12 Objekten ein Proband seine Aufmerksamkeit lenkt, ergab eine Genauigkeit von durchschnittlich 89,3\% ( $\sigma=9,2 \%$ ). Die Dekodierungsgenauigkeit aller Probanden lag deutlich über dem Rateniveau von 8,3\% und bei 7 Probanden sogar über $95 \%$. Mit einem Permutationstest, bei dem wir die Klassifikation 500 mal pro Proband mit zufälliger Zuordnung des Zielreizes zu den Trials wiederholt haben, haben wir außerdem das Konfidenzintervall bestimmt, das angibt, welche Dekodierungsgenauigkeit man mit diesem Klassifikationsschema durch Zufall erreichen kann. Die Analyse ergab, dass die Wahrscheinlichkeit, Dekodierungsgenauigkeiten über $12,7 \%$ zu erreichen, unter $5 \%$ liegt, Dekodierungsgenauigkeiten über $16,5 \%$ zu erreichen, unter $0,1 \%$.

In $>$ Abb. 2 sind beispielhaft die ersten 2 kanonischen Komponenten dargestellt, die hauptsächlich zur Dekodierung der EKPs beitragen. Wir vergleichen die beiden Komponenten mit den Differenzwellen an Cz und PO7. Es fällt auf, dass die ersten beiden kanonischen Komponenten mit der Differenzwelle an Cz, wo typischerweise das P300 Potenzial gemessen wird, und an PO7, wo typischerweise für Diskriminierungsprozesse relevante N1/N2 Komponenten zu finden sind, signifikant korrelieren. Die Komponente mit dem höchsten kanonischen Korrelationskoeffizienten (1. kanonische Komponente) korreliert nicht notwendigerweise mit dem P300 Potenzial. Man beachte, dass die kanonischen Komponenten individuell sehr unterschiedlich aussehen können und hier nur einzelne Beispiele dargestellt sind.

Die hohe Korrelation der Komponenten mit Cz und PO7 suggeriert, dass es hinreichend sein könnte, direkt die Signale an Cz und PO7 als Komponente zu betrachten und mit deren Differenzwelle, die aus einer Trainingsmenge bestimmt wurde, zu korrelieren, um den Zielreiz zu bestimmen. Dies haben wir getestet und haben eine mittlere Dekodierungsgenauigkeit von 56,5\% ( $\sigma=12,9 \%$ ) an Cz, von 47,8 \% ( $\sigma=15,3 \%)$ an PO7 und von 63,1\% ( $\sigma=13,6 \%)$, wenn beide Kanäle als Komponenten involviert sind, erreicht. Alle Ergebnisse unterscheiden sich statistisch signifikant von der erreichten Dekodierungsgenauigkeit mittels optimaler räumlicher Filter. Ein Überblick über die Ergebnisse ist in \ Tab. 1 dargestellt.

\section{Diskussion}

Wir haben hier ein Verfahren vorgestellt, mit dem man aus dem EEG Komponenten extrahieren kann, die den Unterschied zweier Experimentalbedingungen optimal repräsentieren. Dabei lernt der Algorithmus aus einer Menge vieler Messbeispiele, wie die Signale aller Elektroden kombiniert werden müssen. Wir haben gezeigt, dass man mit den so ermittelten räumlichen Filtern effizient Aufmerksamkeitspotenziale detektieren kann, was eine zuverlässige Steuerung eines $\mathrm{BCls}$ erlaubt. Besonders bemerkenswert ist, dass unser Ansatz unabhängig von Augenbewegungen ist und daher für Patientengruppen interessant, die infolge einer Motoneuron- Erkrankung auch Beeinträchtigungen der Augenmuskeln zeigen. Wie in einem Übersichtsartikel über BCls, die unabhängig von Augenbewegungen sind, berichtet wurde, erreichen andere Ansätze oft deutlich geringere Dekodierungsraten [11]. Ein Ansatz, der auf zentral schnell präsentierte, visuelle Stimuli setzt, erreicht zwar eine

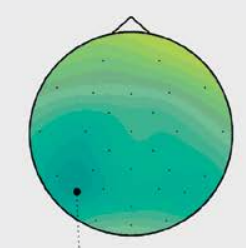

$\mu \mathrm{V}$
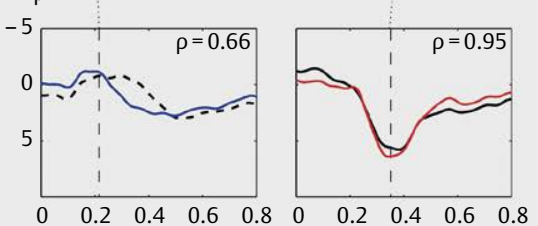
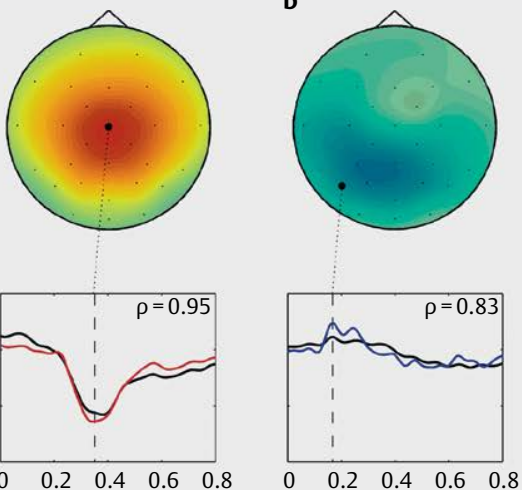
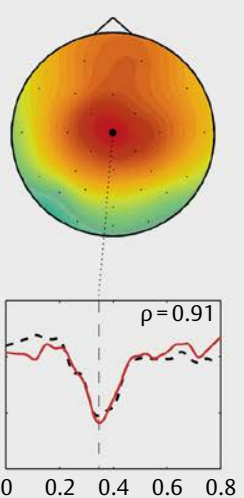
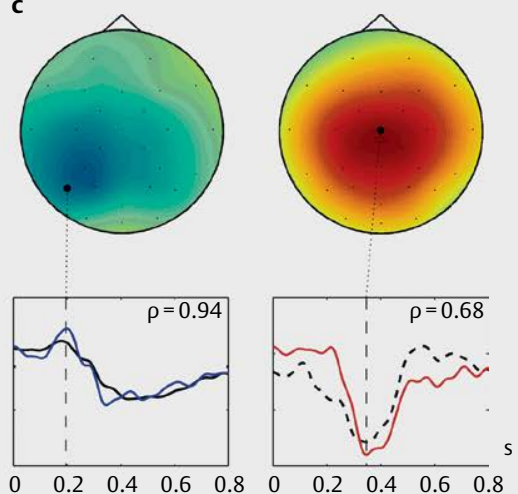

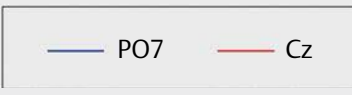

1. kanonische Komponente

- - - 2. kanonische Komponente

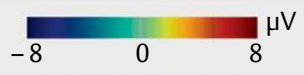

Abb. 2 Die Diagramme zeigen die ersten 2 kanonischen Komponenten und die Differenzwelle zwischen Target- und Standardepochen an PO7 und Cz. Die Korrelationskoeffizienten $\rho$ geben die Produkt-Moment Korrelation zwischen den gezeigten Zeitreihen an. Die Topografien zeigen die Verteilung der Differenzwellen über dem Kopf zu dem Zeitpunkt, der im Diagramm mit einer senkrechten Strichlinie gekennzeichnet ist. a zeigt die Gruppenanalyse, b zeigt einen Probanden mit vergleichsweise geringer Dekodierungsgenauigkeit (70,9\%) und c zeigt einen Probanden mit vergleichsweise hoher Dekodierungsgenauigkeit $(96,1 \%)$. 
- Tab. 1 Im Durchschnitt erreichte Dekodierungsgenauigkeit mit Komponenten aus räumlicher Filterung (mittels CCA) und an einzelnen Standardpositionen.

\begin{tabular}{|l|l|l|l|l|}
\hline Herkunft des EKP & CCA & Cz & P07 & Cz \& PO7 \\
\hline $\begin{array}{l}\text { Mittlere Dekodierungs- } \\
\text { genauigkeit (\%) }\end{array}$ & 89,3 & 56,5 & 47,8 & 63,1 \\
\hline \begin{tabular}{l} 
Standardabweichung \\
\hline
\end{tabular} & 9,2 & 12,9 & 15,3 & 13,6 \\
\hline
\end{tabular}

Erkennungsrate von über $95 \%$, aber die Stimulationszeit ist hier deutlich länger [12]. Nicht nur für betroffene Patienten und deren Angehörige kann ein $\mathrm{BCl}$-basiertes Kommunikationssystem eine wichtige Erleichterung sein, auch Ärzte könnten davon profitieren, Symptomatiken einer Krankheit zu erfahren, die sonst unmöglich mitzuteilen sind.

Mithilfe der relevantesten aus Beispieldaten geschätzten räumlichen Filter konnten wir neue Komponenten extrahieren, deren Verlauf den größten Unterschied der unterliegenden Hirnprozesse kennzeichnet. Es hat sich gezeigt, dass die für die Unterscheidung von Target- und Standardtrials relevantesten Komponenten hohe Korrelationen mit den Differenzwellen an den Standardelektrodenpositionen Cz, mit dem typischen Verlauf des P300 Potenzials, und PO7, mit einer Modulation der N1/N2 zeigt. Das P300 Potenzial, das typischerweise nach Wahrnehmung eines abweichenden Stimulus am Vertex gemessen werden kann, wurde vor mehr als 30 Jahren erstmals für eine Kommunikation mittels $\mathrm{BCl}$ eingesetzt [13]. Die Relevanz der parieto-okzipitalen N1/N2 Komponenten lässt sich nicht nur durch Studien der visuellen Aufmerksamkeit erklären, die diesem Potenzial eine Beteiligung an frühen Selektionsprozessen [14] und Diskriminationsprozessen [15] zuschreiben. Sie wurden auch in anderen P300-basierten BCI Studien als relevantes Merkmal identifiziert $[12,16]$.

Das hier vorgestellte Verfahren ist also in der Lage, selbständig die Elektroden so zu gewichten, dass Unterschiede in den Hirnantworten zweier experimenteller Bedingungen als neue Komponente hervorgehen. Prinzipiell könnte diese Komponete das Signal einer Quelle im Gehirn darstellen, denn auch die Rekonstruktion von Quellen kann als räumlicher Filter betrachtet werden, bei der eine spezielle Montage, also Gewichtung von Kanälen für die Transformation in den Quellenraum verwendet wird [17]. Allerdings werden hier keine anatomischen Gegebenheiten berücksichtigt, sodass jede Komponente auch aus einer Kombination mehrerer Quellen bestehen kann und somit räumlich-zeitlich unterschiedliche Hirnprozesse reflektieren kann. Sofern also nicht die genaue Lokalisation eines Hirnprozesses, sondern die Existenz event-korrelierter Potenziale untersucht werden soll, ist dieses Verfahren durchaus auch für klinische Anwendungen geeignet. Beispielsweise stellt die Dekodierungsgenauigkeit eine einfach interpretierbare Evaluierungsgröße dar, mit der man die Präsenz einer Hirnantwort auf einen Reiz quantitativ bewerten könnte.

\section{KERNBOTSCHAFT}

Mit der hier vorgestellten Methode haben wir gezeigt, wie Gehirnaktivität, die sich als event-korreliertes Potenzial im EEG widerspiegelt, mit statistischen Lernmethoden entschlüsselt werden kann. Die erlernten Zusammenhänge haben wir genutzt, um effizient aus dem EEG die Aufmerksamkeit auf einen in der Peripherie des visuellen Feldes präsentierten, visuellen Stimulus mit hoher Genauigkeit zu erkennen. Unser Ansatz ermöglicht eine von Augenbewegungen unabhängige Steuerung eines $\mathrm{BCls}$, wovon Patienten mit schweren motorischen Defiziten profitieren können. Die datengetriebene Analyse von Gehirnaktivität ist nicht nur bei der Steuerung eines BCls anderen Ansätzen überlegen, man kann damit auch Fragestellungen des klinischen EEGs adressieren.

\section{Danksagung}

Die Arbeit wurde teilweise vom BMBF mit dem Förderkennzeichen 13GW0095D im Forschungscampus STIMULATE finanziert.

\section{Interessenkonflikt}

Die Autorinnen/Autoren geben an, dass kein Interessenkonflikt besteht.

Literatur

[1] Quandt F, Reichert C, Schneider B et al. Grundlagen und Anwendung von Brain-Machine Interfaces (BMI). Klin Neurophysiol 2012; 43 : $158-167$

[2] Lal TN, Schröder M, Hinterberger T et al. Support Vector Channel Selection in BCI. IEEE Trans Biomed Eng 2004; 51: 1003-1010

[3] Cecotti H, Rivet B, Congedo M et al. A Robust Sensor-Selection Method for P300 Brain-Computer Interfaces. J Neural Eng 2011; 8: 016001

[4] Feess D, Krell MM, Metzen JH. Comparison of Sensor Selection Mechanisms for an ERP-based Brain-Computer Interface. PloS one 2013; 8: e67543

[5] Blankertz B, Tomioka R, Lemm S et al. Optimizing Spatial Filters for Robust EEG Single-Trial Analysis. IEEE Signal Process Mag 2008; 25 : $41-56$

[6] Lotte F, Bougrain L, Cichocki A et al. A Review of Classification Algorithms for EEG-Based Brain-Computer Interfaces: a 10 Year Update. J Neural Eng 2018; 15: 031005

[7] Fazel-Rezai R, Allison BZ, Guger C et al. P300 Brain Computer Interface: Current Challenges and Emerging Trends. Front Neuroeng 2012; 5: 14

[8] Reichert C, Dürschmid S, Heinze H-J et al. A Comparative Study on the Detection of Covert Attention in Event-Related EEG and MEG signals to control a BCI. Front Neurosci 2017; 11: 575

[9] Hotelling H. The most predictable criterion. J Educ Psychol 1935; 26: 139

[10] Lin Z, Zhang C, Wu W et al. Frequency Recognition Based on Canonical Correlation Analysis for SSVEP-Based BCls. IEEE Trans Biomed Eng 2006; 53: 2610-2614 
[11] Riccio A, Mattia D, Simione L et al. Eye-Gaze Independent EEG-Based Brain-Computer Interfaces for Communication. J Neural Eng 2012; 9: 045001

[12] Treder MS, Schmidt NM, Blankertz B. Gaze-Independent BrainComputer Interfaces Based on Covert Attention and Feature Attention. J Neural Eng 2011; 8: 066003

[13] Farwell LA, Donchin E. Talking off the Top of your Head: Toward a Mental Prosthesis Utilizing Event-Related Brain Potentials. Electroencephalogr Clin Neurophysiol 1988; 70: 510-523

[14] Hillyard SA, Münte TF. Selective Attention to Color and Location: An Analysis with Event-Related Brain Potentials. Percept Psychophys 1984; 36: $185-198$
[15] Hopf J-M, Vogel E, Woodman G et al. Localizing Visual Discrimination Processes in Time and Space. J Neurophysiol 2002; 88: 2088-2095

[16] Shishkin SL, Ganin IP, Basyul IA et al. N1 Wave in the P300 BCl is not Sensitive to the Physical Characteristics of Stimuli. J Integr Neurosci 2009; 8: 471-485

[17] Scherg M, Ille N, Bornfleth $\mathrm{H}$ et al. Advanced Tools for Digital EEG Review: Virtual Source Montages, Whole-Head Mapping, Correlation, and Phase Analysis. J Clin Neurophysiol 2002; 19: 91-112 